\title{
Variations
}

Variations

Revue internationale de théorie critique

$20 \mid 2017$

Expériences oppositionnelles

\section{Les nouvelles politiques du conflit}

Paola Sedda

\section{(2) OpenEdition}

Journals

Édition électronique

URL : http://journals.openedition.org/variations/825

DOI : 10.4000 /variations.825

ISSN : 1968-3960

Éditeur

Les amis de Variations

Référence électronique

Paola Sedda, «Les nouvelles politiques du conflit », Variations [En ligne], 20 | 2017, mis en ligne le 25 avril 2017, consulté le 25 février 2021. URL : http://journals.openedition.org/variations/825 ; DOI https://doi.org/10.4000/variations.825

Ce document a été généré automatiquement le 25 février 2021.

Les ami•e•s de Variations 


\title{
Les nouvelles politiques du conflit
}

\author{
Paola Sedda
}

\section{Introduction}

1 «Libération de soi », « désertion active », " révolution silencieuse $»^{1}:$ depuis la fin des années 1960 , on assiste à la prolifération de syntagmes originaux ayant la fonction de saisir les nouveaux sens attribués à l'engagement politique. Ainsi, la thèse principale qui s'affirme progressivement défend l'émergence de nouvelles mobilisations, moins idéologiques et non plus plus orientées vers la conquête du pouvoir politique. Les spécialistes ont alors identifié un passage d'une forme de militantisme total et fondé sur l'adhésion idéologique à une nouvelle forme de "militantisme distancié » orienté vers la réalisation de projets spécifiques et régi par des réseaux instables d'activistes². En effet, la grande variété de mouvements sociaux qui se sont développés aux cours des années 1960 et 1970 a marqué le début d'un processus de morcellement progressif des fronts de lutte. Ainsi, en 1980, cette tendance à la diversification des groupes et des revendications (féministes, écologistes, séparatistes, anti-raciales ...) a été interprétée par Alberto Melucci comme le signe d'un renouveau de l'action collective. Les «nouveaux mouvements sociaux » se situeraient donc aux marges du conflit politique et seraient essentiellement engagés dans la promotion de nouvelles identités sociales ${ }^{3}$.

2 À la fin des années 1990, les désastres causés par la globalisation économique et financière déclenchent une nouvelle vague de contestation qui s'oppose aux injustices perpétrées par le marché néolibéral. Ainsi, en dépit du tournant culturaliste identifié par Melucci, les récents mouvements de crise qui se sont érigés contre les élites politiques dans le monde arabe et contre le précariat e les reformes du travail en Europe s'opposent aux dérives de la société de marché. La nature des revendications ne suffit donc pas à décréter l'entrée dans une nouvelle ère de la contestation. S'agit-il alors d'une transformation concernant la composition, l'organisation ou les formes d'engagement ? Les mouvements sociaux qui se sont succédés au cours des dernières décennies (des zapatistes du Chiapas aux Indignés espagnols) semblent en effet proposer 
des nouvelles pratiques de la contestation et des formes originales d'autogestion et de délibération démocratique.

3 En allant à contre-courant par rapport à la théorie de la rupture d'époque ${ }^{4}$, nous faisons ici le pari de la continuité entre les luttes du passé et du présent. Dans cette perspective, les évolutions du militantisme s'inscrivent dans un processus historique où les dimensions socio-politiques et socio-économiques contribuent mutuellement à faire évoluer les dynamiques contestataires. En effet, si les États nationaux cautionnent l'actuelle configuration du marché et leur procurent une consistance politique par le biais des instances internationales, la marge de manœuvre à disposition des acteurs contestataires du conflit politique se réduit progressivement et poussent ces derniers à rechercher les ressources nécessaires pour l'action en dehors du périmètre institutionnel. Ce déplacement semble donc constituer une démarche stratégique consciente au sein des luttes contemporaines. Dans ce même esprit, le mouvement zapatiste incitait à la construction de "nouveaux terrains de lutte » et de nouveaux «sujets subalternes» agissant en dehors de l'État-nation et des formes de la représentation électorale ${ }^{5}$.

4 En accentuant le processus de redéfinition des frontières du politique, cet éloignement des champs d'action traditionnels peut également impliquer une dilution du potentiel contestataire. Le patient, l'usager et le consommateur militant constituent seulement quelques exemples de l'actuelle panoplie de figures de l'empowerment citoyen. Mais la fragmentation des thématiques et des acteurs de la contestation n'a pas pour autant abouti au passage à un régime politique fondé sur la délibération élargie. Au contraire, la possibilité d'un changement politique radical apparaît de plus en plus verrouillée. Derrière l'accélération et l'illusion d'un mouvement permanent, nos sociétés semblent plonger dans un état $\mathrm{d}^{\prime}$ " immobilisme structurel $»^{6}$. Ainsi, si les phénomènes de fragilisation des formes stables et organisées de l'action collective sont indéniables, des nouveaux projets contestataires, plus ou moins durables, continuent à éclore de partout en proposant des formes originales de réappropriation du politique. D'Internet à la rue, l'idée centrale est celle d'expérimenter des nouvelles formes de participation démocratique. La rhétorique de la participation empreigne désormais la quasi totalité des initiatives contestataires et partisanes. Elle peut être instrumentale à l'obtention de la légitimation et de l'adhésion électorale (comme dans le cadre des campagnes présidentielles), constituer une ressource organisationnelle pour la réalisation d'un projet politique plus stable (comme dans le cas du Mouvement 5 étoiles en Italie), ou, encore, elle peut être vécue comme un horizon de lutte (comme dans le cas du mouvement Nuit Debout). Le déficit démocratique émerge donc dans sa forme pathologique que l'on essaie de soigner par le biais d'incursions ponctuelles et efficacement médiatisées dans l'espace public.

5 Nous allons alors essayer de saisir les changements qui traversent la sphère contestataire en nous concentrant, plus particulièrement, sur l'impact des usages sociaux d'Internet dans l'évolution des formes organisationnelles et des pratiques militantes. En proposant une critique de la «théorie des nouveaux mouvements sociaux", nous allons réhabiliter le cadre d'analyse du conflit politique où les dynamiques contestataires sont appréhendées au prisme des modèles organisationnels et des équilibres changeants de la polity ${ }^{7}$. Ce paradigme doit aujourd'hui pouvoir dialoguer avec les études de la communication électronique jouant un rôle de plus en plus important dans la formation des opinions politiques, la construction des nouveaux 
cadres contestataires et l'organisation de l'action collective. Il s'agira alors d'abandonner la dichotomie douteuse entre stratégie et identité pour réaffirmer la pertinence d'une perspective fondée sur le conflit politique et en saisir les possibilités d'actualisation. Les «nouvelles politiques du conflit " se nourrissent des pratiques et des lieux émergeants de la communication sociale étant à la base de la formation des "espaces publics oppositionnels». Le recours aux approches critiques de l'espace public $^{8}$ nous permettra de développer une réflexion autour des démarches, militantes et intellectuelles, visant à réinvestir le concept d' « espace public » en lui restituant la dimension conflictuelle qui lui est propre.

\section{L'organisation contestataire aujourd'hui}

\section{Technologie et organisation}

6 L'évolution de la sociologie des mouvements sociaux marque le passage d'une approche psychologique à une approche structurelle centrée sur le concept d'organisation et d'action stratégique. Ce déplacement conceptuel débute au sein de l'École de Chicago avec les études sur le «comportement collectif» et s'accomplit avec le travail de l'économiste Mancur Olson', considéré généralement comme l'initiateur du paradigme de la mobilisation des ressources. Ce dernier préconise que la constitution d'une organisation, présentant des spécificités propres au champ contestataire, permet à un groupe de se structurer et de rassembler les ressources nécessaires à la mobilisation.

Qu'elle s'affiche en tant que structure professionnelle ou citoyenne, verticale ou horizontale, formelle ou informelle, reposant sur des ressources informationnelles ou matérielles, l'organisation est une dimension essentielle de l'action collective ainsi qu'une condition pour sa réussite et sa continuité dans le temps. La plupart des sociologues de la mobilisation semblent appuyer cette affirmation. Selon Gamson, ce sont les mouvements les plus hautement organisés et bureaucratisés à avoir montré un degré majeur d'efficacité dans l'impact social de leurs revendications et dans la réalisation des résultats préfigurés ${ }^{10}$. Plus attentif au niveau d'intégration sociale des groupes contestataires, Anthony Oberschall affirme que tout conflit social est lié aux inégalités de position, de classe ou de statut et se déclenche donc à partir des conflits d'intérêt concernant les différents groupes sociaux ${ }^{11}$. La proximité ou la distance du pouvoir politique détermineraient le niveau de radicalisation du mouvement (qui augmente proportionnellement à l'augmentation de la distance du pouvoir) ainsi que le type d'action menée. Même dans le modèle du conflit politique élaboré par Charles Tilly, l'organisation, dotée d'une identité commune et de réseaux internes, est considérée comme un facteur déterminant pour le succès de la mobilisation ${ }^{12}$. En s'appuyant sur le modèle développé par Anthony Oberschall, Tilly identifie des groupes de «challengers" (des statuts socio-professionnels ou des groupes spécifiques de citoyens disposant d'un potentiel mobilisateur majeur par rapport à d'autres) et affirme l'importance des variables sociales dans la constitution de l'organisation. Celleci dépend de la rencontre entre les identités liées aux catégories objectives (les ouvriers, les étudiants ou les femmes, par exemple), et la création de réseaux volontaires de la part des agents sociaux.

Le processus de création des liens et des réseaux organisationnels semble avoir été bousculé par l'arrivée du numérique. En effet, la généralisation des usages d'Internet 
permet de gérer des ressources collectives en autonomie et à un coût très limité et de maximiser et élargir les possibilités et les modalités de diffusion, de partage et de coproduction de l'information militante. Les réseaux des «liens faibles " s'ajoutent aux réseaux des liens identitaires forts en favorisant la multiplication des groupes d'intérêt et de cause ainsi qu'une gestion autonome et collaborative de leur médiatisation. Le réseau des activistes s'articule alors aux autres réseaux d'internautes en impliquant une diversification des manières dont on acquiert le capital social de l'organisation. La maitrise des outils numériques favorise donc l'émancipation des activistes des organisations professionnelles et contribue à faire évoluer les compétences et les savoirs militants qui sont progressivement assimilés aux pratiques communicationnelles ordinaires. Ainsi, la création d'espaces info-communicationnels alternatifs, produisant un contre-agenda médiatique et capables d'auto-fabriquer l'image et le sens véhiculé par les mouvements, exprime cette volonté de défier la pensée dominante en en manipulant les codes et les formats de communication. Les pratiques communicationnelles assument donc une importance croissante dans toutes les phases de la mobilisation : la création de l'identité contestataire, le choix de la forme organisationnelle, le recrutement et la coordination de l'action collective.

\section{La «tyrannie de l'horizontalité ${ }^{13}$}

9 Au début des années 1970, la féministe Joe Freeman nous mettait en garde sur les risques dérivés de "l'idéologie de l'absence de structure " qui commençait à se répandre dans les milieux contestataires. En l'absence d'une modalité efficace pour diffuser les informations et partager les savoirs militants, la rhétorique de l'horizontalité peut freiner la participation et les contributions des militantes les plus démunies se référant à un cadre d'action bien délimité ${ }^{14}$.

10 Souvent considérées comme intrinsèquement innovantes, les formes organisationnelles des mouvements sociaux revêtent une fonction à la fois stratégique et expressive. La complémentarité de ces deux dimensions implique une homologie importante entre les aspects organisationnels et les valeurs portées par les activistes. Réfractaires au fonctionnement hiérarchisé et vertical, assimilable aux organisations partisanes traditionnelles, les mouvements sociaux sont tout de même appelés à se structurer afin de produire et valoriser leurs ressources collectives.

11 Les mouvements sociaux contemporains déclarent s'appuyer sur une organisation souple, horizontale et anti-autoritaire qui semble identifier, dans le réseau Internet, une base technique idéale. D'un point de vue stratégique et organisationnel, l'architecture et l'éthique d'Internet semblent encourager la décentralisation et la coopération. Toutefois, ces évolutions ne sont pas exemptes de contradictions internes. La centralité de l'outil numérique implique aussi une réflexion autour des nouvelles compétences pragmatiques, cognitives et expressives que ce type d'organisation demande aux participants. Comme il a été souligné par différentes études, Internet pourraient creuser encore plus les inégalités dans la participation démocratique ${ }^{15}$. En effet, tout en ayant certainement contribué à réduire les coûts de la circulation de l'information, l'usage d'Internet est devenu aussi un nouveau marqueur de légitimité et de visibilité sociale. Charles Tilly remarque à ce propos que la coordination par moyens électroniques favorise les pays riches et sur-sélectionne les élites militantes des pays pauvres ${ }^{16}$. Il est donc important de souligner que si Internet peut encourager l'adoption 
de modalités originales de l'action collective, il n'efface pas pour autant les relations de pouvoir. Au contraire, le poids des affinités et des compétences expressives fait reposer toute la vie du groupe sur l'activité d'un petit réseau d'usagers hyper-actifs en suscitant donc parfois des hiérarchies encore plus marquées.

12 En ce sens, malgré la force et l'importance revêtue par la rhétorique de l'horizontalité dans la phase de création de l'identité collective, celle-ci constitue un horizon commun d'attente plus qu'une réalité effective. Internet favorise l'individualisation des pratiques militantes et la centralité des activités informationnelles et de gestion des audiences en ligne dans le processus de mobilisation mais il ne modifie pas la stratification du tissu militant ni la composition des figures et des degrés d'engagement. La mobilisation numérique repose en effet sur un nombre restreint d' " architectes» de la contestation ${ }^{17}$, tandis que de nombreux activistes assument plutôt une fonction de passeur, de diffuseur ou d'amplificateur de la mobilisation numérique. Ces derniers jouent un rôle variable dans le processus de construction de la conscience protestataire qui dépend avant tout du temps, des ressources et du niveau d'engagement de chaque adhérent ${ }^{18}$. Certains militants se limitent à publier des informations et à les diffuser auprès de leurs réseaux des connaissances. D'autres adhèrent à des initiatives locales, contribuent à créer des collectifs et à disséminer l'action dans les territoires. D'autres encore se font transporter par l'effet de contagion du like.

13 Une illustration intéressante du fonctionnement de la mobilisation numérique est constituée par le mouvement italien Il popolo viola (Le Peuple violet) ayant été à l'origine d'une grande manifestation populaire, le NoBerlusconiDay, qui s'est déroulée en décembre 2009 à Rome et dans d'autres villes à l'étranger. Il s'agit d'un cas plutôt atypique car la mobilisation, ayant pour but de demander la démission du président Silvio Berlusconi, démarra avec la création d'un groupe satyrique sur Facebook de la part d'une poignée de blogueurs. L'indignation de ce petit groupe éveilla très vite l'intérêt de la toile. Le groupe reçut ainsi des centaines de milliers de pouces levés. Devant tant de succès et d'adhésion, ce qui n'était au départ que l'expression d'un rasle-bol se transforma vite en mouvement organisé actif, coordonnant les ressources collectives en ligne et sur les territoires et diffusant l'information militante. En inaugurant une nouvelle pratique militante à coût zéro, le contenu de la page Facebook du collectif reflétait le formatage de l'information qui caractérise ce réseau social. La proposition de sortir de Facebook pour défiler dans les rues, de donner un corps et un visage à cette démocratie protestataire, généra beaucoup d'enthousiasme et se traduisit par la mobilisation de nombreux groupes qui se sont coordonnés en ligne et sur le terrain afin de récolter les fonds, diffuser l'information, organiser les transports et la logistique ${ }^{19}$.

14 Ainsi, l'organisation des groupes au niveau local et la diffusion virale d'informations militantes pour inciter les internautes à s'exprimer et à participer semblent représenter les traits principaux de ce nouveau modèle d'organisation. Au-delà de la mobilisation numérique, l'idée de passer à l'acte a largement contribué à accroître la popularité du collectif et à multiplier les adhésions. L'action de terrain reste donc une étape essentielle pour donner une dimension pleinement politique aux pratiques numériques. 


\section{Faut-il avoir peur de l'organisation ?}

15 «Une fois que le mouvement aura cessé de s'accrocher à l'idéologie de l'absence de structure, il aura la possibilité de développer les formes d'organisation qui seront davantage en accord avec son fonctionnement $»^{20}$. La tension vers l'absence de structure traverse une bonne partie des initiatives contestataires actuelles. Le cas du collectif italien du Peuple violet et de son processus de démobilisation précoce nous incite à réfléchir sur l'émergence d'une sorte de refus de la structure. En effet, cette forme d'action passagère, qui a caractérisé également le récent mouvement français Nuit Debout, est peut-être due à une volonté précise de ne pas transformer à terme le mouvement en une structure verticale. Mis à part quelques porte-paroles, il est souvent difficile d'identifier la structure organisationnelle des mouvements contemporains. Ainsi, le maintien d'une organisation horizontale et participative semble représenter l'essence même des nouveaux mouvements oppositionnels. Dans l'expérience du Peuple violet, le respect de l'organisation participative et de la délibération horizontale devient toutefois une sorte de prison conceptuelle qui lui empêche de forger des nouvelles catégories politiques et d'avoir un réel impact sur le processus de prise de décision. Le Peuple Violet est donc emblématique d'une nouvelle forme de militantisme où « l'action prévaut sur l'idée de l'action ${ }^{21}$.

Un exemple d'autre nature est constitué par le Movimento 5 stelle (le Mouvement 5 étoiles), l'organisation politique liée au blog de Beppe Grillo. La particularité de cet espace informationnel est constituée par sa double facette : il fonctionne à la fois comme plateforme de contre-information et d'échange informationnel et comme outil de mobilisation et d'action politique. Né pour stimuler la participation politique des citoyens, ce projet a montré sa capacité à animer et élargir le débat public et à faire coïncider l'action informationnelle et militante sur le web avec la mobilisation de terrain. En effet, le comique a réussi à faire converger le désenchantement d'une large partie de citoyens vers la création d'un nouveau projet politique qui a élu la sphère d'Internet comme principal lieu de socialisation et d'élaboration de l'action collective. Plus qu'un mouvement, il s'agit désormais d'une véritable organisation numérique et partisane qui se projette dans l'avenir et qui vise la conquête du pouvoir politique. Ici la rhétorique de la participation citoyenne et de l'horizontalité coexiste, d'une manière surprenante, avec une organisation stable, hautement formalisée et incarnée par un leader charismatique. Toutefois, malgré les différences, les mécanismes de mobilisation des 5 étoiles manifestent des similitudes avec ceux développés par le Peuple violet. Dans les deux cas, la dynamique collective est née de la rencontre d'un ensemble de pratiques communicationnelles individuelles, jusque-là dispersées dans le réseau. La mobilisation peut donc être le résultat d'une rencontre entre des individus dispersés qui partageaient une opinion politique mais qui avaient besoin d'un cadre communicationnel pour l'exprimer et agir collectivement.

17 La différence réside donc plutôt dans la fonction expressive revêtue par l'organisation dans les deux mouvements. Les 5 étoiles ont eu progressivement besoin d'assurer la création d'une organisation formelle, avec un statut ${ }^{22}$, un manifeste, un programme et des normes plutôt rigides que les membres et les élus sont appelés à respecter, peine l'exclusion immédiate du mouvement (formellement sujette au vote en ligne). Ces normes sont censées être garantes de la transparence et de la moralité du mouvement. L'observation de l'activité du mouvement a démontré qu'Internet joue un rôle central 
dans la phase d'adhésion (à travers la modalité d'inscription en ligne), de consultation (à travers l'accès aux contenus sur le blog), de délibération (à travers le forum, les sondages et la participation au débat du blog) et d'élection politique (par le biais du vote numérique). L'inscription à la communauté politique est gratuite, s'effectue directement en ligne et elle est ouverte à tous les internautes majeurs qui n'ont pas adhéré par ailleurs à d'autres organisations politiques considérées comme incompatibles avec les principes du mouvement. Les militants ont la possibilité de s'exprimer librement, d'utiliser la plateforme pour organiser des événements et se mobiliser sur le plan local et national. De fortes doutes restent toutefois quant au fonctionnement des formes de délibération et d'élection politique en ligne. Malgré les nombreux points d'intérêt du projet, la participation semble se réduire souvent au vote des internautes sur des propositions dont le processus de sélection reste peu transparent. Comment faire émerger des projets communs et des pratiques de délibération égalitaires de la forme communicationnelle du blog ? Comment le statut, le programme et les normes internes du collectif sont-ils définis à partir des échanges balbutiants de la toile?

Le problème ici n'est pas tant celui de la solution technique qui pourrait, potentiellement, se plier et découler d'un principe démocratique ${ }^{23}$, quant celui de l'absence d'une orientation politique commune. La recherche de l'horizontalité comme fin en soi ou l'expression d'une méfiance vis-à-vis de toute forme d'organisation structurée ne constituent donc pas en elles-mêmes un rempart contre l'autoritarisme. La voie vers une forme de participation élargie doit être bâtie à travers un processus collectif de délibération duquel devrait émerger l'organisation de l'action collective et de ses principes. Dans cette perspective, les normes et les rôles de chacun devraient être le produit du débat et du vécu partagé de l'expérience militante. Les activités d'autogestion et de préservation du commun ne dépendent donc pas tellement de la dimension technique mais elles relèvent plutôt des formes d'élaboration et d'élection des normes qui régissent l'action ${ }^{24}$. Nous nous retrouvons donc dans une situation paradoxale : alors que les techniques à disposition nous donnent accès à des formes de collaboration collective inédites, nous agissons en tâtonnant, en l'absence d'un universel politique qui puisse faire converger les multiples actions de résistance, aujourd'hui inter-catégorielles et internationales, face aux abus des classes dominantes. De ce principe politique, découleraient ensuite des solutions algorithmiques, pensées et conçues à partir de cette même éthique égalitaire de la collaboration mutuelle. Au lieu de ça, nous faisons face, au contraire, au risque d'un aplatissement des formes de militantisme au modèle de l'économie numérique ${ }^{25}$. Dans une logique proche de celle de l'entreprise post-fordiste, l'action militante est orientée au développement de projets spécifiques et axée sur les performances individuelles ${ }^{26}$. La spécificité du capitalisme contemporain consiste précisément en sa capacité à englober et à intégrer la critique en anéantissant son essence propre et son effet ${ }^{27}$. La présence d'un substratum idéologique commun entre la littérature du management et les nouvelles valeurs de la critique sociale nous déplace vers un terrain visqueux. Les concepts de réseau, d'autogestion, de coopération horizontale ou d'organisation par projets caractérisent à la fois le fonctionnement et les valeurs affichées par la nouvelle économie et les réseaux qui militent contre son avancée aveugle. 


\section{Les nouvelles politiques du conflit}

\section{Du conflit politique à la politique du conflit}

19 La disponibilité des ressources collectives et la possibilité de les organiser et de les valoriser d'une manière stratégique sont des présupposés nécessaires mais pas suffisants pour assurer le déclenchement et la réussite des phénomènes contestataires. Nous nécessitons alors d'une approche contextuelle de l'étude des mouvements sociaux qui prenne en compte le processus politique et les dynamiques conflictuelles des sociétés. En effet, les deux terrains que nous avons présentés plus haut, ne se situent pas dans un vide politique. Issus tous les deux du macro-cadre contestataire de l'antiberlusconisme, ils partagent une même frustration vis-à-vis de l'incapacité de la classe politique à garantir et sauvegarder les principes démocratiques. Ces mouvements se sont également développés en coïncidence avec une période de crise économique mondiale et un climat politique marqué par les scandales qui ont concerné l'ensemble des partis traditionnels.

Qu'ils soient directement engagés dans la conquête du pouvoir ou plutôt orientés vers l'expérimentation de nouvelles formes de débat public et de délibération citoyenne, les mouvements contemporains constituent encore des acteurs centraux du conflit politique. Le modèle du «conflit politique » a été systématisé dans l'œuvre de Charles Tilly $^{28}$. En revendiquant une forte attache au matérialisme de Marx et à l'utilitarisme de John Stuart Mill, Tilly conçoit l'action collective dans les termes d'un conflit entre des groupes qui défendent des intérêts communs et qui luttent pour accéder au pouvoir politique. Il combine ainsi deux approches : celle du modèle politique, désignant les phénomènes de conflit des groupes qui luttent pour la conquête du pouvoir politique, et celle du modèle de la mobilisation, centrée sur l'analyse de l'organisation des ressources collectives et des relations politiques d'un mouvement ${ }^{29}$. Le mouvement social doit donc être conçu comme une forme historique particulière de la "politique du conflit» qui se définit par la présence d'une campagne durable de revendications qui utilise des représentations répétées pour se faire connaître du grand public (pétition, manifestations, slogans, interfaces numériques etc ...), qui valorise des ressources communes (argent, travail, armes ou information) et qui s'appuie sur une base $^{30}$. L'action de la base, se déployant en relation avec l'environnement historique et politique, résulte de la rencontre de la structure organisationnelle avec l'univers des croyances du groupe en question. L'action collective d'un groupe est donc une fonction de l'extension des intérêts partagés et de l'efficacité de son organisation et de sa mobilisation $^{31}$. Le groupe, limité par la structure des opportunités politiques, mobilise donc des ressources (elles mêmes limitées) dans le but d'obtenir des biens communs. La rencontre des variables liées à la mobilisation avec le contexte des opportunités politiques déterminerait donc les gains et les pertes liés à l'action collective.

Afin d'aboutir à une vision globale des dynamiques de l'action collective, le modèle de la mobilisation que nous venons de décrire doit dialoguer avec le modèle politique s'intéressant aux relations que les groupes entretiennent avec le gouvernement et les autres acteurs impliqués dans le conflit. Le rapport au pouvoir n'est pas considéré dans les termes durkheimiens du contrôle social mais il est conçu comme un modèle dynamique des opportunités où les acteurs collectifs ont la possibilité d'agir pour modifier les équilibres politiques. Les relations que le mouvement entretient avec les 
autres acteurs politiques se manifestent sous des formes variables qui vont de la répression à la facilitation et de l'opportunité à la menace ${ }^{32}$. En ligne générale, l'entrée de nouveaux membres de la polity implique une retombée positive sur l'accès généralisé à des nouveaux droits et, plus en général, sur l'action collective même ${ }^{33}$. Toutefois, l'évaluation de la réussite d'un groupe peut se révéler une opération très compliquée. En effet, l'accès du mouvement à la sphère politique n'implique pas forcement son succès ni la réalisation de ses objectifs. Bien au contraire, le processus d'institutionnalisation pourrait provoquer le déplacement et le renoncement aux idéaux originels ainsi que la perte conséquente de la base.

Les groupes protestataires assument donc des formes historiques et culturelles changeantes qui, en partant des soulèvements paysans, prennent la forme de l'émeute urbaine ou celle plus structurée du mouvement ouvrier. L'analyse menée par Tilly sur l'évolution des formes conflictuelles en Europe occidentale aboutit à l'identification de trois formes historiques de l'action collective : l'action compétitive, prédominante au cours $d u \mathrm{XV}^{\mathrm{e}}$ et $\mathrm{du} \mathrm{XVI}^{\mathrm{e}}$ siècle et désignant une lutte pour la défense d'intérêts parallèlement revendiqués par un groupe rival; l'action défensive ou réactive, prédominante dans la période comprise entre le XVII et le $\mathrm{XIX}^{\mathrm{e}}$ siècle et indiquant une réaction d'un groupe à une attaque, à une privation ou à la menace d'un droit (comme par exemple dans la lutte pour l'expropriation des latifundia en Italie du Sud); et, enfin, la forme proactive, devenue prédominante à partir du $\mathrm{XX}^{\mathrm{e}}$ siècle et désignant l'émergence de nouvelles revendications, comme dans le cas des mouvements de grève pour l'obtention de meilleures conditions de travail ${ }^{34}$. L'aboutissement à cette forme historique de la politique du conflit correspond à trois processus de changement : le passage des ressources des organisations de petite échelle aux états nationaux et aux agents du marché international (1600 et 1850) ; la nouvelle gestion des ressources qui s'en suit et, enfin, au cours du XIX ${ }^{e}$ et du XX ${ }^{e}$ siècle, l'essor des nouveaux moyens de communication accompagnant la généralisation des systèmes démocratiques. Les nouvelles technologies de l'information et de la communication impliquent une baisse des coûts de la mobilisation liée aux phénomènes de masse et assument un rôle d'accélérateur du processus d'évolution de l'action collective ${ }^{35}$.

\section{L'innovation technologique des répertoires d'action}

23 Tout en admettant la présence d'une grande variété de types de conflit, les chercheurs identifient deux éléments récurrents dans le modèle de la "politique du conflit » : les représentations, des modalités standardisées auxquelles les acteurs font appel (la manifestations, la grève etc...) et le répertoire, l'ensemble des représentations préexistantes et disponibles dans un contexte spatio-temporel donné ${ }^{36}$. Les variations dans les représentations s'opèrent par le biais de choix innovateurs de la part des mouvements et concernent souvent une ré-contextualisation ou un usage atypique d'un outil classique. Le mouvement anti-esclavagiste, par exemple, a contribué à transformer l'outil de la pétition, jadis consistant en un instrument à usage individuel, en un moyen de revendication de masse souvent concernant des personnes différentes des signataires.

Aujourd'hui, l'une des principales innovations des mécanismes contestataires semblent être constituée par l'adoption des outils numériques de la part des nouveaux collectifs. Ces modalités de l'action militante sont conçues par les auteurs en tant que 
représentations modulaires car, dotées d'une grande flexibilité et ductilité, peuvent constamment être adaptées et modifiées à partir du contexte local et des objectifs spécifiques du groupe. L'innovation technologique des répertoires représente donc un thème de réflexion central dans l'étude des mouvements contemporains. Les auteurs soulignent que, malgré la présence de répertoires de coutume et d'une certaine régularité dans les représentations adoptées par les parties du conflit (comme il a été montré par le couple ouvriers-patrons), le répertoire n'implique pas l'absence d'improvisation. Tarrow et Tilly présentent une typologie des répertoires qui va des répertoires faibles, des représentations en voie de définition dans des contextes de transition politique, aux répertoires forts, pouvant constituer un ensemble de représentations rituelles et institutionnalisées (comme dans le cas des manifestations du premier mai).

Bien qu'il soit important de ne pas confondre les répertoires de contestation (qui, pour Tilly, ont évolué dans l'espace de trois siècles) avec les répertoires tactiques et les innovations des performances déterminées par l'entrée de l'hacktivisme, les outils numériques se diffusent à une rapidité déconcertante en entraînant des changements importants dans les modalités d'engagement et de participation des militants. Les nouveaux outils comme l'e-mailing, les newsletters, les blogs, les wikis ou la communication via les principaux réseaux sociaux sont devenus des moyens incontournables de l'action collective. Ces outils assument une importance croissante non seulement en tant que stratégies de sensibilisation de l'opinion publique et de recrutement des nouveaux adhérents, mais surtout en tant que nouvelles modalités d'organisation et de socialisation politique. Dans cette perspective, les outils du web social contribuent à restructurer et reconfigurer les formes communicationnelles et organisationnelles et à déterminer l'émergence de nouvelles compétences et modes d'action au sein du champ militant. Plus qu'un medium, Internet peut être conçu comme un espace public relativement libre, moins sujet au contrôle politique et permettant l'adoption de formes alternatives de participation et d'organisation. Interprété en tant que nouvelle forme sociale, Internet « concourt sur des modes inédits à la constitution des communautés ». Le lien social n'est plus conçu sous la forme d'une agrégation, d'une identification ou d'une coordination mais il devient " pure circulation $»^{37}$. Conçu en tant que «forme politique», Internet a alimenté depuis ses débuts la perspective d'une intervention plus directe des citoyens dans la chose publique. Dans l'environnement d'Internet, la production et la valorisation des ressources des mouvements impliquent toujours un travail collaboratif où l'engagement individuel ne demande pas des dépenses excessives et cela soit en termes économiques, soit au niveau de l'énergie et du temps consacrés au projet. La gestion verticale des professionnels de la communication éclate donc face à des nouvelles formes d'engagement, moins contraignantes et plus individualisées. Ici la connaissance des outils numériques et la disponibilité d'audiences diversifiées constituées par les contacts des réseaux numériques représentent des nouveaux critères déterminant l'impact et la participation de chacun à l'action collective.

26 Ces observations sur les nouveaux enjeux d'Internet n'impliquent pas une disparition automatique des répertoires et des formes contestataires classiques. Au contraire, la manifestation et la grève restent les moyens principaux de l'action protestataire au sein de catégories spécifiques comme celles des syndicats ou des travailleurs. Toutefois, à côté des performances classiques, le recours aux représentations numériques indique souvent une volonté d'innover le champ de l'action militante. Puisque les modes 
d'action peuvent se lire comme des «technologies de mobilisation et de représentation $»^{38}$, la pratique contestataire en ligne est à concevoir aussi comme un choix idéologique, comme une marque de fabrique de certains groupes se reconnaissant dans l'esprit libertaire et décentralisateur d'Internet. En effet, au-delà des avantages concernant la baisse des coûts de l'action collective, l'usage résistant d'Internet implique également l'adhésion à une nouvelle éthique de l'horizontalité et de la participation caractérisant la plupart des mouvements contemporains. Ces constats ne nous amènent pas à décréter une rupture nette avec les modes d'engagement du passé mais plutôt une évolution historique et progressive des tactiques et des représentations de la contestation dont les moteurs sont à rechercher à la fois dans les mutations socio-politiques et socio-techniques. Dans cette optique, nous considérons que la prégnance historique de la notion de «nouveau mouvement social » n'est pas automatiquement acquise. La plupart des mouvements contemporains constituent en effet des formes de militantisme hybrides qui combinent les performances contestataires traditionnelles avec des nouveaux modes d'action et de mobilisation numérique.

\section{Le conflit politique est aussi un conflit culturel}

Il va de soi qu'Internet ne doit pas être considéré uniquement comme un espace organisationnel et un outil stratégique mais également comme un nouveau terrain voué à l'expression, la socialisation et la construction identitaire.

Ainsi, même dans l'environnement numérique, la dimension culturelle des différents groupes sociaux demeure centrale. L'existence d'inégalités sociales et structurelles et la disponibilité de ressources pour l'action ne suffisent donc pas à déclencher et à expliquer le phénomène de la mobilisation sociale. Pour que les acteurs décident de passer à l'acte, ils doivent avoir construit une représentation partagée de l'injustice sociale.

29 La perspective culturaliste et cognitiviste dans l'analyse de la mobilisation s'inscrit dans un processus de renouvellement des études sur les mouvements sociaux qui démarre au début des années 1980. En réhabilitant l'apport de l'interactionnisme symbolique de l'École de Chicago, l'approche cognitiviste de la mobilisation repose essentiellement sur l'héritage goffmanien de l'analyse des cadres de l'expérience. Selon Erving Goffman, le cadre « permet à son utilisateur de situer, de percevoir, d'identifier, de nommer un nombre quasiment infini d'occurrences concrètes définies en ses termes ${ }^{39}$. Si, d'un côté, le modèle de Goffman nous invite à considérer l'existence de cadres idéologiques hégémoniques qui sont sélectionnés et activés par les individus, de l'autre côté, il nous incite également à penser que l'activité contestataire consiste en la création de nouvelles représentations politiques produites en opposition aux cadres officiels. En ce sens, le concept de cadre doit être conçu non seulement comme la forme principale à travers laquelle s'exerce le contrôle social sur les consciences mais également dans sa dimension conflictuelle qui est intrinsèque au processus même de création et de consolidation des croyances. Dès lors, le conflit politique doit être interprété également comme un conflit culturel, c'est-à-dire en tant que lutte pour l'affirmation d'un cadre idéologique aux dépenses de celui des opposants. Dans cette perspective, la nouvelle dichotomie conceptuelle qui distingue les mouvements stratégiques des mouvements identitaires ${ }^{40}$, et qui reflète le fossé qui s'est 
progressivement creusé entre les tenants $d u$ modèle du conflit politique et les défenseurs de la théorie des nouveaux mouvements sociaux, apparait comme une fausse opposition. Cette distinction entre les deux types de mouvement n'est en effet pas si nette et de nombreux groupes ont tendance à combiner la forme expressiveidentitaire avec celle stratégique-instrumentale ${ }^{41}$. Certaines analyses comparatives conduites à ce sujet montrent que les mouvements identitaires ne sont pas si fréquents et que la plupart des actions contestataires est portée par des collectifs citoyens qui protestent contre les grands projets publics ou industriels. Les combats concernant la construction de l'aéroport de Notre-Dame-des-Landes en France ou la ligne ferroviaire à haute vitesse entre Turin et Lyon en Italie sont représentatifs de ce type de conflit politique. En n'étant pas en opposition, identité et intérêt constituent bien plutôt une dyade stratégique qui était également présente dans le modèle du mouvement ouvrier du XIXème siècle. Ici les revendications salariales des ouvriers étaient indissociables de la quête de la reconnaissance de leur dignité et de leur identitée ${ }^{42}$. Cette dernière contribue en effet à définir le champ et les dynamiques conflictuelles à l'intérieur de l'espace social.

30 C'est précisément au niveau de ce processus d'appropriation du cadre culturel que les pratiques info-communicationnelles jouent un rôle essentiel. Dans l'environnement numérique, le processus discursif de construction de sens est primordial pour définir une identité collective et stimuler la participation. Le déclenchement d'une mobilisation numérique dépend donc aussi de la constitution d'une vision politique forte qui est partagée par une masse critique d'internautes. Afin de pouvoir constituer une identité contestataire, il est donc nécessaire de toucher un certain nombre d'usagers qui seront disponibles à participer activement au débat et à produire des contenus en ligne. L'activation et l'intensification des pratiques infocommunicationnelles autour d'un projet collectif sont donc à considérer comme des ressources fondamentales pour la naissance et l'aboutissement du projet militant.

31 La question que l'on se pose alors est celle de savoir si nous assistons à un processus de fragmentation et de multiplication des cadres contestataires ou alors s'il est possible de faire dégager un horizon culturel commun qui relierait les nouveaux projets militants. L'observation de l'Internet contestataire italien montre que les nouvelles pratiques militantes s'inscrivent dans une démarche idéologique libertaire et anti-parti qui, à notre sens, doit être considérée comme un processus de réappropriation locale de mouvances culturelles plus vastes. La participation est ici considérée comme un antidote à une crise de la démocratie représentative et du modèle organisationnel $\mathrm{du}$ parti politique. À l'image du " parti cartel », ce dernier serait désormais déconnecté des territoires et des contextes de vie réels et uniquement orienté vers l'auto-préservation. La solution apportée par les mouvements contemporains consiste alors dans la constitution d'un non-parti citoyen où le débat démocratique doit être ouvert et égalitaire et se dérouler en l'absence de toute forme de médiation. Ces mouvements prétendent souvent se situer dans un univers post-idéologique où, aux clivages politiques de la droite et de la gauche, on superpose un processus de confrontation d'idées censées défendre l'intérêt collectif. Le cadre idéologique des nouveaux mouvements se construit donc progressivement autour du dépassement du concept de démocratie représentative. Les processus de délégation et de représentation devraient alors laisser la place à un système permettant aux citoyens de rentrer directement dans les institutions et d'y rester pour une durée limitée. 
L'adhésion au mouvement se produit par le biais de la contribution individuelle au processus de production du cadre contestataire. En ce sens, l'échange discursif et la production de contenus en ligne n'ont pas uniquement une finalité stratégique et vouée au recrutement mais relèvent d'une nouvelle approche de l'engagement politique. Au lieu d'un univers préétabli sur lequel le militant devrait s'aligner, le cadre contestataire est donc conçu comme un terrain fluide que les échanges info-communicationnels contribuent constamment à définir. Les changements du militantisme ne sont donc pas à rechercher dans la nature des revendications, définies comme plus culturelles, sociétales ou identitaires, ni dans la disparition du modèle du conflit politique, mais plutôt dans le déplacement et l'évolution des lieux et des modalités de la socialisation politique.

\section{Pratiques de la communication et contestation}

\section{Communication électronique et action collective}

Il devient de plus en plus urgent de s'interroger, dans une perspective critique, sur le rôle joué par les pratiques numériques dans les processus de construction des identités collectives et dans l'évolution des formes organisationnelles. De ces questionnements, en découlent naturellement d'autres concernant l'émergence des nouvelles arènes et des nouvelles formes de débat politique en ligne. Pour ce faire, il convient tout d'abord de prendre ses distances de toute approche déterministe : il va de soi que l'accès à une dotation technique n'implique pas automatiquement un usage civique et engagé de celle-ci. Dans la plupart des cas, les militants numériques correspondent à ceux qui disposent d'un capital symbolique important et qui sont déjà intéressés par la politique en dehors des sphères de la communication électronique ${ }^{43}$. La technologie n'a bien évidemment pas le pouvoir intrinsèque de résoudre le déficit de participation démocratique. Elle peut par contre favoriser, auprès des groupes de citoyens actifs, l'émancipation des structures professionnelles et institutionnelles. En tant qu'accélérateur des changements en cours, les innovations techniques peuvent accentuer le phénomène déjà mentionné de de-institutionnalisation de la contestation sociale.

En raison de la diffusion et de la circulation des nouvelles pratiques et des nouveaux savoirs militants, nous avons donc besoin de reconsidérer le concept d'« organisation contestataire » et les activités annexes de construction et de valorisation des ressources collectives. À notre sens, les innovations ne concernent pas tellement le déplacement de la mobilisation des espaces physiques aux espaces virtuels mais résident plutôt dans l'importance croissante que les pratiques numériques revêtent dans l'ensemble des phases de la mobilisation sociale : formation de l'identité collective, construction du cadre contestataire, recrutement des nouveaux adhérents, organisation et coordination de l'action.

L'autre aspect que nous devrions prendre en considération est l'incidence de la catégorie du temps dans les processus de mobilisation. Dans la littérature anglosaxonne, de Blumer à Tilly, les mouvements sociaux sont caractérisés par une certaine continuité dans le temps. Ce critère semble avoir été mis à mal par les principes de l'accélération ${ }^{44}$ impliquant une variation constante des pratiques, des croyances et des engagements pris par les acteurs sociaux. Cela signifie aussi, qu'au-delà de la 
《structure des opportunités politiques ${ }^{45}$, d'autres aspects, considérés comme étant conjoncturels (l'actualité politique et économique, les situations de crise financière; l'économie des médias nationaux et internationaux) ont la capacité de déclencher une mobilisation rapide au sein de groupes sociaux dont les revendications et les motivations étaient restées jusque-là dormantes. Trop rigide, le modèle de «la structure des opportunités politiques" ne considère pas non plus la capacité des contestataires à se créer eux mêmes des occasions pour l'action. Comme il a été souligné à juste titre par Lilian Mathieu, l'ouverture des institutions ou l'élection d'un parti politique dont les principes sont proches du mouvement contestataire pourrait avoir l'effet contradictoire d'affaiblir la mobilisation ${ }^{46}$. Mais le cas contraire est aussi possible : dans le cadre de la récente mobilisation française contre la « loi travail» (loi El Khomri), la déception provoquée par la démarche ambiguë d'une force politique théoriquement proche des idées du mouvement (en l'occurrence, le Parti Socialiste Français) a enclenché un processus de radicalisation et d'intensification du combat.

Il émerge alors la nécessité de prendre en compte les sphères de la communication dans l'appréhension des phénomènes de l'action collective. En effet, il ne suffit pas que des opportunités politiques se présentent, pour que les acteurs puissent les saisir, il faut également que les processus de socialisation politique et de médiatisation du dissensus soient murs. Les mouvements sont en effet de plus en plus liés à des écosystèmes informationnels spécifiques favorisant la constitution de publics critiques éclatés. En correspondance avec des conjonctures historiques et politiques particulières, ceux-ci arrivent à percer dans l'espace public. Cependant, les mutations socio-techniques que nous avons décrites ne se produisent pas d'une manière homogène au sein des différents groupes sociaux. Dans la plupart des mouvements, les interactions en face-à-face revêtent encore une fonction primordiale dans la constitution de l'identité collective. Dans d'autres cas, les nouvelles formes de communication numérique sont cruciales dans la construction de l'identité du groupe et l'organisation de l'action militante. Nous défendons donc ici la thèse d'une coprésence entre l'action collective traditionnelle, fondée sur la création de ressources collectives et organisationnelles stables, et l'action connective (" connective action »), basée sur des rencontres et des engagements ponctuels qui sont issus des réseaux d'intérêts et des activités communicationnelles en ligne ${ }^{47}$. Dans cette perspective, la construction de l'identité collective doit être considérée comme un processus lent et diffus qui ne s'accomplit pas uniquement au sein des mouvements mais qui doit être recherché en amont et en aval de l'action collective ainsi que dans la variété des sphères et des formes de la communication sociale. Le mouvement social ne constitue donc pas uniquement une forme de production et d'organisation des ressources collectives mais il est le lieu où des idées et des conflictualités latentes deviennent conscientes et assument une consistance politique.

\section{Espaces médiatiques oppositionnels et mobilisation}

L'élaboration d'une théorie critique de la communication est souvent associée au travail du philosophe allemand Jürgen Habermas. Selon Habermas, suite au déclin du mouvement ouvrier, de nouveaux conflits émergent dans les contextes structurés par la communication. Ces derniers seraient indépendants des sphères de la reproduction matérielle et des organismes institutionnels. Dans son modèle, le système d'indemnisation et de rationalisation de l'État social, accompagné par un processus 
d'individualisation des relations de travail, aurait encouragé un refoulement des structures de classe. Dès lors, l'aliénation se manifesterait avant tout dans sa dimension politique et interviendrait dans les contextes de formation de l'opinion publique ${ }^{48}$. Face au caractère immanent des sociétés néo-libérales et du système de marché, les seules formes de résistance viables consisteraient ainsi, pour le philosophe, dans la création d'espaces autonomes de délibération. Il s'agirait alors d'un pouvoir communicationnel qui, tout en n'ayant pas accès au pouvoir décisionnel, peut l'influencer et stimuler un processus de changement politique et social.

Or, malgré sa présumée portée critique, le modèle de démocratie délibérative reposant sur l'idéal normatif de la sphère publique bourgeoise n'est guère convaincant. En proposant de détacher l'espace public des contextes de vie et d'action des citoyens, Habermas contribue à désamorcer le potentiel humain de rébellion. En effet, la distinction nette entre espace privé, professionnel et public apparaît problématique car c'est précisément au cours du processus contestataire que l'on pourra forger le débat public et que l'on décidera des thèmes de préoccupation commune. Si la publicité est définie par le processus contestataire, sa relation avec la position sociale devient donc bien plus complexe que celle que Habermas nous laisse entendre. Selon le philosophe, des personnes privées, faisant un usage public de leur raison, devraient suspendre leurs intérêts personnels en nom du bien commun ${ }^{49}$. Mais cela implique une adhésion à des formes de discussion et à des principes qui peuvent exclure certaines catégories sociales et contribuer, paradoxalement, à reproduire les inégalités. En effet, comme il a été suggéré par Nancy Fraser, il ne suffit pas d'affirmer qu'une arène de discussion doit être un espace où les distinctions sociales existantes sont suspendues et neutralisées pour qu'il en soit ainsi ${ }^{50}$. Selon cette auteur, nous avons assisté depuis toujours à la formation et à l'action de contre-publics (les mouvements nationalistes, les ouvriers, les femmes ...) qui entretenaient des rapports conflictuels avec les publics bourgeois ${ }^{51}$. En refusant la dichotomie qui oppose le terrain des luttes de classe aux luttes identitaires et liées à la reconnaissance, Nancy Fraser affirme que la volonté de valoriser les différences, par le biais d'une transformation des valeurs culturelles hégémoniques, ne devrait pas s'opposer aux luttes pour l'obtention d'un système politique et économique plus égalitaire. La subordination économique interdit, en effet, toute participation à la production culturelle, dont les normes sont elles-mêmes institutionnalisées par l'État et par le monde économique ${ }^{52}$. En ce sens, nous pouvons affirmer qu'il ne peut pas y avoir une politique de la différence sans l'application préalable d'une politique universaliste de l'égalité.

La plupart des mouvements contemporains est aujourd'hui engagée dans la construction d'espaces de communication autonomes voués à favoriser la construction d'une identité collective et à gérer la représentation symbolique et médiatique du projet contestataire. Nous pouvons mentionner à ce propos le rôle du blog de Beppe Grillo dans la construction de la communauté politique des 5 étoiles ${ }^{53}$ ou, bien avant, la création du réseau d'information Indymedia dans le cadre des luttes altermondialistes. La communication, dans la multiplicité de ses canaux et de ses formats, constitue donc une sphère privilégiée tant pour l'affirmation de la pensée hégémonique que pour la construction de formes de résistance symbolique.

40 À l'instar d'Oskar Negt ${ }^{54}$, nous pouvons considérer ces zones d'information et de communication citoyennes comme étant des "espaces médiatiques oppositionnels $»^{55}$. En prolongeant l'élan radical de la Théorie critique, Negt affirme la nécessité d'assurer 
la constitution d'un "espace public oppositionnel », à caractère plébéien, capable de fédérer les expériences vécues et de canaliser les problèmes substantiels d'aujourd'hui. Ceux-ci n'apparaissent que de façon superficielle dans l'espace public bourgeois tel qu'il se manifeste ${ }^{56}$. En animant un débat critique avec Habermas, le concept d' «espace public oppositionnel » nous permet d'analyser les prises de parole d'acteurs qui ne sont pas reconnus comme légitimes à l'intérieur de l'espace public politique. Ce dernier constituerait, selon Habermas, « le concept fondamental d'une théorie normative de la démocratie $\aleph^{57}$. En réalité, le processus discursif de formation de l'opinion politique tel qu'il a été décrit par Habermas comporte l'exclusion d'une vaste partie de citoyens. Ainsi, la multiplication des lieux et des formes d'expression dans l'espace public, encouragée, entre autre, par la généralisation des usages d'Internet ${ }^{58}$, offre des nouvelles opportunités de prise de parole et de construction du politique. Les « espaces médiatiques oppositionnels " émergeants sont porteurs de discours et de pratiques propres qui se distinguent à la fois des protocoles de la politique institutionnelle et des formes de militantisme traditionnel. Ces sphères sont en effet vouées à donner une expression politique aux aspirations démocratiques des différentes catégories de la société ${ }^{59}$.

41 En démentant la théorie selon laquelle les mouvements sociaux ne seraient plus intéressés à participer au processus politique, les collectifs contemporains se démontrent capables de défier les appareils des partis et d'élaborer des nouvelles formes de débat et de délibération originales. En ce sens, le sentiment «antipolitique ", partagé par une grande partie des groupes contestataires, a été interprété à tort comme un éloignement du politique. En réalité, en adressant directement leurs critiques aux sphères du pouvoir médiatique et politique, les nouveaux collectifs essaient de désavouer la démocratie représentative et de créer les bases pour une participation civique élargie. Il se dessine donc progressivement une nouvelle figure de militant qui, de plus en plus sceptique vis-à-vis des modalités traditionnelles de l'action politique, se concentre sur la gestion critique de l'information et sur l'animation d'« espaces médiatiques oppositionnels ». Ces pratiques de communication résistantes vont acquérir une consistance plus proprement politique à travers l'action complémentaire menée sur les territoires. Comme il a été confirmé par les cas des Indignés en Espagne ou du Mouvement 5 étoiles en Italie, l'imbrication des sphères en ligne et hors ligne se révèle être un élément essentiel pour la réussite de la mobilisation et la continuité du projet contestataire.

42 Dans le contexte de cette nouvelle politique du conflit, la construction de la critique sociale militante se nourrit de la création d' « espaces médiatiques oppositionnels » qui se superposent à l'ordre symbolique dominant ${ }^{60}$. Ainsi, comme l'espace public oppositionnel d'Oskar Negt permet l'expression d'une démocratie rebelle, déconnectée des normes qui régissent l'espace public bourgeois ${ }^{61}$, de la même façon, les pratiques émancipatrices qui se développent dans les environnements numériques permettent aux activistes de déserter les lieux de pouvoir pour se forger, en marge, une conscience protestataire.

\section{Conclusion}

43 La faillite des états communistes, l'affirmation de la "gauche molle » dans les démocraties occidentales, l'affaiblissement progressif des organisations ouvrières et, 
enfin, la montée de la xénophobie et du nationalisme, semblent indiquer l'impossibilité d'un monde au-delà du capitalisme. Ce dernier continue en effet à déployer partout sa force implacable tout en démontrant son incapacité à apporter des solutions aux crises et aux désastres que lui même a créés ${ }^{62}$. C'est dans cet espace vide qu'émergent les nouvelles politiques $d u$ conflit. Elles sont donc à la fois le produit et la réaction au long processus historique ayant amené à l'affirmation idéologique du modèle de la démocratie libérale. Dans ce contexte, l'idée de révolution s'estompe ne dessinant plus un impératif d'ordre stratégique. En effet, au cours de l'histoire, l'idée de Marx d'une société qui devrait se définir par sa capacité d'auto-organisation égalitaire a été supplantée par l'instauration de ce que Victor Serge a défini dans les termes d'un «monopole bureaucratique et policier $"^{63}$. La crise de la gauche historique marque alors la victoire discursive du libéralisme en tant que pouvoir autocratique transnational. En même temps, l'échec politique de l'État-nation dans la défense des intérêts collectifs a contribué à repousser les possibilités d'invention du politique au-delà des canaux traditionnellement voués au combat social. À partir de la fin des années 1990, les mouvements progressistes considèrent en effet l'État comme un engrenage asservi au système économique global. Face à cette force autocratique, les militants s'engagent dans la récupération d'un « espace public pour tout le monde qui ne correspond pas à la prise de pouvoir d'un petit groupe ni à la création de nouveaux appareils et de nouvelles bureaucraties $»^{64}$.

Ainsi, tandis que le potentiel révolutionnaire, conçu dans les termes d'une confrontation directe entre les classes sociales aboutissant à leur destruction, s'estompe, il émerge un nouveau potentiel de rébellion essentiellement fondé sur les principes de renforcement de la démocratie. Le champ contestataire devient alors un laboratoire de la citoyenneté. Les devises « Democracia real ya » du mouvement du 15Mai (les Indignés) et le "Democracy Now » de Occupy Wall Street démontrent la construction transfrontalière de cet imaginaire de lutte commun. En Europe et ailleurs, les nouvelles politiques du conflit constituent donc des variantes locales d'une même lutte contre le libéralisme économique et la précarisation existentielle qui en découle. Dans le cadre de ces nouvelles expériences contestataires, l'usage civique des plateformes numériques, permettant au citoyen de s'exprimer dans l'espace public, représente une étape importante dans la réalisation de cet idéal de démocratie radicale. Ce glissement, du terrain politique orthodoxe à un nouveau terrain promouvant une pratique forte de la citoyenneté, n'indique pas, toutefois, l'absence d'implications politiques. Ces mouvements oppositionnels proposent une critique solide de la logique des partis et des failles de la démocratie représentative. Cela les pousse à repenser les espaces et les formes de la participation afin de combler la distance et la défiance qui se sont créées au fil du temps vis-à-vis des élites.

Les discours de la critique sociale militante semblent faire écho à certains principes de la Théorie critique. Selon Oskar Negt, les bases d'une démocratie radicale sont indissociables de la création d'un contexte public et militant dans lequel toutes les femmes et tous les hommes peuvent donner une expression politique à leurs intérêts et à leurs aspirations ${ }^{65}$. Cet espace peut dépasser le stade oppositionnel et favoriser l'élaboration de nouvelles formes de délibération et d'action politique destinées à renouveler, ou même à se substituer, à l'arsenal de l'espace public bourgeois. Toutefois, ces changements ne peuvent pas se réaliser en dehors du principe de solidarité humaine et de juste partage des biens qui, comme le précise Negt, " ne doit pas être 
réduit à une question éthique ou à une sorte de bonne volonté, mais doit être formulé en termes politiques ${ }^{66}$. Aujourd'hui, les nouvelles technologies facilitent et accélèrent le passage à l'action. La contestation assume ainsi des modalités très variées qui vont des concerts aux pétitions en ligne, des hashtags aux débats et rassemblements via les réseaux sociaux. Mais ces actions, disséminées dans le temps et dans l'espace, ne peuvent pas être considérées comme un horizon ultime. L'invention du politique doit pouvoir reposer sur l'organisation et la mobilisation d'une base populaire qui agit d'une manière stratégique et qui laisse son empreinte dans l'Histoire.

\section{NOTES}

1. On fait ici référence à un certain nombre de travaux qui soulignent le basculement de la lutte stratégique, visant la prise du pouvoir, vers d'autres formes de combat finalisées à libérer l'individu ou à stimuler un changement des valeurs sociales par le biais de ce que Inglehart a défini comme étant une « révolution silencieuse ». Parmi les auteurs qui se sont intéressés à ce phénomène, nous pouvons mentionner Hakim Bey et son utopie pirate ainsi que Michael Hardt et Tony Negri prônant l'idée d'une dissémination d'actes de résistance finalisés à déserter l'empire et à en dévoiler l'aspect parasitaire.

The silent revolution: Changing values and political styles among Western publics, Princeton Legacy Library ; Hakim Bey., (2002), TAZ : Zone autonome temporaire, Paris, l'Éclat ; Hardt M. et Negri A., (2000), Empire, Harvard : Harvard University Press;), ; Inglehart R., (1977).

2. Ion J., (1997), La fin des militants ?, Paris : Éditions de l'Atelier.

L'expression "militantisme distancié », utilisée par Jacques Ion pour saisir les transformations qui traversent ce champ, constitue un constat sociologique de départ qui nous permet d'approcher un terrain politique plus sociétal, moins marqué par les clivages traditionnels et fondé sur l'utilisation des plateformes collaboratives en ligne.

3. Melucci A., (1980), "The new social movements : A theoretical approach», Social Science Information, $\mathrm{n}^{\circ} 19$, p. 199-226.

4. Castells M., (2001), L'ère de l'information, tome 1, La société en réseau, Paris : Fayard. De nombreux travaux sociologiques cautionnent une théorie de la rupture d'époque. En Sciences de l'information et de la communication, la théorie la plus aboutie semble être celle de Manuel Castells. Le sociologue appuie l'idée de l'émergence de la "société informationnelle» dans laquelle les réseaux informatiques, en tant que principale source de productivité et de pouvoir, constitueraient « la nouvelle base matérielle de la société ».

5. EZLN (1996), Crónicas Intergalácticas, Premier Encuentro Intercontinental por la Humanidad y contra el Neoliberalismo, Chiapas, México, « EZLN, B. Como se resiste el poder global », p. 45-47.

6. Rosa H., (2010), Accélération, Une critique sociale du temps, Paris : La Découverte.

7. Tilly C., (1976), From Mobilization to Revolution, Reading, MA : Addison-Wesley.

8. Fraser N. (2005), Qu'est-ce que la justice sociale ?, La Découverte ; Negt O., (2007), L'espace public oppositionnel, L'expérience plébéienne, une histoire discontinue de la liberté politique, Paris : Payot.

9. Olson M., (1978), Logique de l'action collective, Paris : PUF.

10. Gamson W. A., (1975), The Strategy of Social Protest, Belmont : Wadsworth Pub.

11. Oberschall A., (1973), Social Conflict and Social Movements, Englewood Cliffs : Prentice Hall, p. 33.

12. Tilly,1976, op. cit. 
13. Freeman J., (1974), "The Tyranny of Structurelessness ", In Jane S. Jaquette, ed., Women in Politics, New York: Wiley. Traduction française du titre de l'essai de Joe Freeman, activiste nordaméricaine $\mathrm{du}$ mouvement pour la libération des femmes (http://1libertaire.free.fr/ JFreeman01.html).

14. Ibid.

15. Granjon F., (2009), «Inégalités numériques et reconnaissance sociale. Des usages populaires de l'informatique connectée ", Les Cahiers du numérique, 2009/1 (Vol. 5), p. 19-44 ; George É., (2008), « De la complexité des relations entre démocratie et TIC », Nouvelles pratiques sociales, vol. 21, $\mathrm{n}^{\circ} 1, \mathrm{pp} .38-51$.

16. Tilly C., (2004), Social Movements 1768-2004, Boulder \& London : Paradigm Publishers.

17. Oberschall, 1973, op. cit.

18. George, 2008, op. cit.

19. Sedda P., (2015b), «La politisation de l'ordinaire: enjeux et limites de la mobilisation numérique ", Sciences de la Société, n 94, Médias, Engagements, Mouvements sociaux, n 94, 2015, p. 157-175.

20. Freeman, 1974, op. cit.

21. Ion J., Franguiadakis S. et Viot P., (2005), Militer aujourd'hui, Paris : Éditions Autrement.

22. Le Mouvement 5 étoiles se définit comme une non-association, il refuse toute forme de structure et de hiérarchie et il ne semble pas épouser une forme organisationnelle précise. En effet, bien que le blogueur soit indéniablement le leader et dicte la ligne politique du mouvement, il n'y a pas de coordination verticale à proprement dit. Les listes civiques se constituent d'une manière indépendante, elles sont certifiées à distance et, dans la plupart des cas, les activistes locaux ne connaissent pas personnellement leur leader. Le non-statut peut être consulté à la page http://www.beppegrillo.it/listeciviche/liste/arcidosso/non-statuto-del-movimento-5-stelle-diarcidosso.html.

23. Feenberg A., (2014), Pour une théorie critique de la technique, Lux Éditeurs, Montréal.

24. Dardot P. et Laval C. (2014), Commun, Essai sur la révolution au XX siècle, Paris, La Découverte.

25. Casilli, Antonio A. (2015) «Digital labor : travail, technologies et conflictualités », in D. Cardon \& A. A. Casilli, Qu'est-ce que le digital labor ?, Bry-sur-Marne, INA, coll. « Etudes et controverses ».

26. Granjon F., (2001), L'Internet militant, Mouvement social et usages des réseaux télématiques, Rennes : Editions Apogée.

27. Boltanski L. e Chiapello E., (1999), Le nouvel esprit du capitalisme, Paris, Gallimard.

28. Tilly, 1976, op. cit.

29. Ibid., p. 3.

30. Tilly C. et Tarrow S., (2008), Politique(s) du conflit, De la grève à la révolution, Paris : SciencePo Les Presses.

31. Tilly, 1976, op. cit. p. 50.

32. La répression peut être orientée vers l'action collective elle-même (comme dans le cas de la répression policière) ou vers la mobilisation (comme dans le cas des activités de répression subies par le Parti Communiste américain). La réaction du gouvernement, qui varie en fonction du type de système politique (répressif, totalitaire, tolérant ou faible), dépend aussi des caractéristiques de l'action et de son niveau d'acceptabilité. Plus en particulier, selon Tilly, dans les mobilisations à grande échelle la tendance répressive est majeure. Les groupes qui bénéficient d'un certain pouvoir seront à l'inverse plus facilement tolérés ou favoris.

33. Tilly, 1976, op. cit. p. 41-42.

34. Tilly, 1976, op. cit. p. 2-10.

35. Tilly, 1976, op. cit. p. 10.

36. Tilly et Tarrow, 2008, op. cit.

37. Rosanvallon P., (2006), La contre-démocratie, La politique à l'âge de la défiance, Paris : Seuil, p. 73. 
38. Sommier I., Fillieule O. et Agrikoliansky É., dirigé par, (2008), Généalogie des mouvements altermondialistes en Europe. Une perspective comparée, Paris/Aix-en-Provence : Karthala/IEP.

39. Goffman E., (1974), Les cadres de l'expériences, Paris : Éditions Minuit, p. 21.

40. Cohen J. L., (1985), «Strategy or Identity: New Theoretical Paradigms and Contemporary Social Movements ", in Social Research: An International Quarterly Social Movements, Arien Mack, Editor, vol. 53, n¹, pp. 663-716.

41. Kriesi H. , Koopmans R. ; Duyvendak J. W., Giugni M. G., (1995), New Social Movements in Western Europe, Minneapolis : University of Minnesota Press.

42. McAdam, Tarrow et Tilly, 1998, op. cit.

43. George, 2008 op. cit. ; Vedel. T., (2006), «La révolution ne sera plus télévisée. Internet, Information et Démocratie », Pouvoirs, 119/2006.

44. Rosa, 2010, op.cit.

45. Tilly et Tarrow, 2008, op. cit. Ce modèle théorique, élaboré par (considère le niveau d'ouverture et de fermeture des institutions politiques, le degré de stabilité et d'instabilité des alliances politiques et gouvernementales et la présence de conflits entre les élites comme des variables déterminantes pour le développement de l'action contestataire. En ce sens, une configuration politique donnée peut favoriser ou décourager la formation de groupes contestataires et avoir un impact dans le développement et le succès de leur action. Ce modèle trop mécanique ne prend pas en compte la capacité des mouvements à subvertir les règles et les principes démocratiques.

46. Mathieu L., (2002), «Rapport au politique, dimensions cognitives et perspectives pragmatiques dans l'analyse des mouvements sociaux » in Revue française de science politique, 52e année, $n^{\circ} 1$, p. 75-100.

47. Lance Bennett W. \& Segerberg A., (2012), "The Logic of Connective action», Information, Communication \& Society, 15:5, 739-768.

48. Habermas J., (1987), Théorie de l'agir communicationnel, Tome 2, Rationalité de l'agir et rationalisation de la société, Paris, Payard, 1987. Le système bureaucratique et commercial colonise et désamorce les structures communicationnelles résistantes venant du " monde vécu ", lieu constitutif de la personnalité, de la culture et de l'identité sociale.

49. Habermas, J., (1993), L'espace public, Paris : Payot.

50. Fraser N., (1992), « Repenser la sphère publique : une contribution à la critique de la démocratie telle qu'elle existe réellement » in Craig Calhoun (eds), Habermas and the Public Sphere, Mit Press, p. 109-142.

51. Fraser, 1992, op. cit.

52. Fraser N., (2012), «Opposer lutte des classes et revendication de la différence ? Égalité, identité et justice sociale », in Le Monde diplomatique, juin 2012.

53. Sedda P. (2015b), «Espaces numériques oppositionnels et mobilisation en Italie, Communication, Technologie et Développement, TIC et Mobilisations, $\mathrm{n}^{\circ}$ 2, novembre 2015, p. 53-64.

54. Negt O., (2007), L'espace public oppositionnel, L'expérience plébéienne, une histoire discontinue de la liberté politique, Paris, Payot.

55. Sedda P., (2015a), « L'Internet contestataire comme pratique d'émancipation : des médias alternatifs à la mobilisation numérique », Les Cahiers du numérique, 4/2015 vol. 11, p. 25-52.

56. Negt, 2007, op. cit.

57. Habermas, 1993, op. cit. Préface de l'auteur, XXV.

58. Miège B., (2010), Espace Public Contemporain, Grenoble : PUG.

59. Negt, 2007, op. cit., p. 38.

60. Sedda, 2015a, op. cit.

61. Sagradini L., (2009), "La plèbe entre dans la surface de jeu ", Multitudes, vol. 4, n 39, p. 205-210. 
62. Dardot et Laval, op. cit.

63. V. Serge, L'An I de la révolution russe, La Découverte, Paris, 1997, « Postface inédite : Trente ans après (1947) », p. 468, cité par Dardot et Laval, op. cit., p. 61.

64. EZLN, 1996, op. cit., p. 43-44.

65. Negt, 2007, op. cit.

66. Negt, 2007, op. cit., p. 29.

INDEX

Mots-clés : politique du conflit, engagement, action collective, usages contestataires d'Internet, espace public numérique

\section{AUTEUR}

\section{PAOLA SEDDA}

Maître de conférences en sciences de la communication à l'Université de Bourgogne. Ses recherches portent sur les usages contestataires des nouvelles technologies et les nouvelles formes de la citoyenneté 